\title{
Reversible impairment of the ku80 gene by a recyclable marker in Aspergillus aculeatus
}

\author{
Shuji Tani ${ }^{1}$, Atsushi Tsuji ${ }^{1,2}$, Emi Kunitake', Jun-ichi Sumitani ${ }^{1}$ and Takashi Kawaguchi ${ }^{1 *}$
}

\begin{abstract}
Auxotrophic mutants of Aspergillus can be isolated in the presence of counter-selective compounds, but the process is laborious. We developed a method to enable reversible impairment of the ku80 gene (Aaku80) in the imperfect fungus Aspergillus aculeatus. Aaku80 was replaced with a selection marker, orotidine 5'-phosphate decarboxylase (pyrG), followed by excision of pyrG between direct repeats (DR) to yield the Aaku80 deletion mutant (MR12). The gene-targeting efficiency at the ornithine carbamoyltransferase (argB) locus was drastically elevated from 3\% to $96 \%$ in MR12. The frequency of marker recycling depended on DR length. One uridine auxotroph was obtained from $3.3 \times 10^{5}, 1.4 \times 10^{5}$, and $9.2 \times 10^{3}$ conidia from strains harboring 20-, 98-, and 495-bp DRs, respectively. Because these strains maintained the short DRs after $5 \mathrm{~d}$ of cultivation, we investigated whether Aaku80 function was disrupted by pyrG insertion with the 20-bp DR and restored after excision of pyrG. The Aaku80 disruption mutant (coku80) was bred by inserting pyrG sandwiched between 20-bp DRs into the second intron of Aaku80, followed by excision of pyrG between the DRs to yield the coku80rec strain. Analyses of homologous recombination frequency and methyl methanesulfonate sensitivity demonstrated that Aaku80 function was disrupted in coku80 but restored in coku80rec. Furthermore, pyrG was maintained in coku80 at least for ten generations. These data indicated that reversible impairment of ku80 in A. aculeatus is useful for functional genomics in cases where genetic segregation is not feasible.
\end{abstract}

Keywords: Aspergillus aculeatus, Homologous recombination, Ku gene, Counter selection, Marker recycling

\section{Introduction}

Aspergillus aculeatus [NBRC 108796], a filamentous fungus isolated from the soil, produces a number of extracellular cellulose- and hemicellulose-degrading enzymes that exhibit synergism with Trichoderma cellulases. Alkali-treated rice straw was shown to be hydrolyzed almost completely into monosaccharides by mixed-culture filtrate of these two species (Murao et al. 1979, 1988). Previously, we constructed a host-vector system of $A$. aculeatus that expressed the $A$. aculeatus $\beta$-mannosidase gene (Kanamasa et al. 2003). The A. aculeatus expression system yielded a 10-fold higher level of the enzyme than did an Aspergillus oryzae expression system, indicating the high potential of $A$. aculeatus as a factory to produce useful proteins (Kanamasa et al. 2007). However, the concerted action of several cellulases, each with different

\footnotetext{
* Correspondence: takashi@biochem.osakafu-u.ac.jp

'Graduate School of Life and Environmental Sciences, Osaka Prefecture

University, 1-1 Gakuen-cho, Naka-ku, Sakai, Osaka 599-8531, Japan

Full list of author information is available at the end of the article
}

properties, is considered to be essential for the effective hydrolysis of cellulose into monosaccharides. To breed $A$. aculeatus that simultaneously produces many types of cellulases, a marker-recycling technique, enabling to excise a selection marker gene from transformants without leaving any exogenous DNA fragments, must be developed (Akada et al. 2006). This technique enables the excision of a selection marker gene from transformants without leaving exogenous DNA fragments; multiple cellulase genes can be introduced into a host strain with a limited number of selection markers. Furthermore, elucidating how the expressions of the cellulose-degrading enzyme-encoding genes are regulated will improve enzyme production. Therefore, one must develop highly-efficient homologous recombination (HR) techniques as well as a markerrecycling technique in the imperfect fungus $A$. aculeatus.

Recently, deletion of the $k u 70 / 80$ or lig4 genes, which are both involved in DNA double strand repair, was demonstrated to elevate the frequency of HR in filamentous fungi (Ishibashi et al. 2006; Krappmann et al. 2006; 
Mizutani et al. 2008; Ninomiya et al. 2004; Takahashi et al. 2006). These achievements enabled the study of the cellular roles of interesting genes. Based on this information, we cloned and replaced the $A$. aculeatus $k u 80$ gene with a selection marker to yield the $A$. aculeatus $k u 80$ deletion mutant. We also investigated the length of the direct repeats (DRs) required for efficient marker recycling in the $A$. aculeatus ku80 deletion mutant to enable a reversible impairment of $k u 80$.

\section{Materials and methods Strains}

All $A$. aculeatus strains used in this study were derived from wild-type $A$. aculeatus no. F-50. Unless otherwise stated, all strains were propagated at $30^{\circ} \mathrm{C}$ in appropriately supplemented minimal medium (MM) (Adachi et al. 2009). The pyrG (orotidine 5'-phosphate decarboxylase gene)deficient mutants (uridine auxotrophs) were spontaneously isolated by spreading $1 \times 10^{6} \mathrm{~A}$. aculeatus wild-type conidia on a plate containing $1 \mathrm{mM} \mathrm{5-fluoroorotic} \mathrm{acid} \mathrm{(5-FOA)}$ and $0.4 \mathrm{mM}$ uridine. Colonies that showed a 5-FOAresistant phenotype were isolated and transformed with the Aspergillus nidulans pyrG gene (AnpyrG), and strains that showed uridine prototrophy were regarded as $p y r G-$ deficient mutants (pyrG13, uridine auxotrophs), which were used as hosts to breed arginine prototrophs and the ku80 deletion mutant in A. aculeatus.

\section{Nucleic acid analysis}

PCR was performed using PrimeSTAR HS DNA polymerase (TaKaRa, Kyoto, Japan) as described in the manufacturer's instructions, except that the annealing temperature was $55^{\circ} \mathrm{C}$ and there were 30 PCR cycles. All primers used in this study are shown in Table 1. Genomic DNA was isolated from ground mycelia with Micro Smash MS-100R (Tomy, Tokyo, Japan) as described previously (Adachi et al. 2009). Southern hybridization was performed as described previously (Adachi et al. 2009).

\section{Cloning the $A$. aculeatus ku80 and ornithine carbamoyltransferase genes}

A part of the A. aculeatus ku80 gene (Aaku80) was amplified from the $A$. aculeatus genome by PCR using the primers ku80F1 and ku80R3, which were designed to anneal to the region conserved among the Aspergillus ku80 genes. The 1.2-kb PCR product was inserted into the EcoRV site of pBluescript II KS (+) (pBS II KS (+)) (Stratagene, La Jolla, CA, USA), and both strands were sequenced twice. Southern blot analysis of the A. aculeatus genome using the Aaku80 segment as a DNA probe suggested that a 6-kb genomic DNA fragment digested with SphI possessed the Aaku80 gene. This fragment was then cloned into the SphI site of pUC118 (Takara) to yield pAaku80.

\section{Table 1 List of primers used in this study}

$$
\begin{aligned}
& \text { Primers used to clone the Aaku80 segment } \\
& \text { ku80F1 5'-ATGGCNGANAAGGAAGCAACNGT-3' } \\
& \text { ku80R3 5'-CGTCCATAYTCATANCCYTTGGC-3' }
\end{aligned}
$$

Primers used for the A. nidulans pyrG insertion into Aaku80

coku80NF 5'-TAGATATCAGCTCTTCTTGCCATCGC-3'

coku80NR 5'-TTAGCGGCCGCTGAAACCATCAGTCTTGTCTGC-3'

coku80CF 5'-GCCTTAATTAAGAGTCCCACGAAGCGATG-3'

coku80CR 5'-TGCTCTAGATGTCGTCATCTTGATAGCGA-3'

copyrF 5'-TAGCGGCCGCAATGCTCTCTATC-3'

copyrR 5'-CCTTAATTAACCGTTACACATTTCCACTCA-3'

Primers used for the AaargB disruption

AaargB5F 5'-GGCGTGATTCTTTGCTCC-3'

AaargB5r2 5'-GAGAGCATTGTCTGCGGGGTCGGCGTCAGGTC-3'

AaargB3f3 5'-GAGTGGAAATGTGTAACGGATTGCATGGACCCGAG-3'

AaargB3r 5'-GGGGACATGGCTTCCTAC-3'

npyrF3 5'-GACCTGACGCCGACCCCGCAGACAATGCTCTC-3'

npyrR3 5'-CTCGGGTCCATGCAATATGCTGGCTGCAATAAAC-3'

mrarg5F 5'-GGCGGGCTGGAGCTCAC-3'

mrarg5iR 5'-GCACCGCGCTAGCCGGTCATTCG-3'

mrarg5iF 5'-CGAATGACCGGATAGCGCGGTGC-3'

mrarg5R 5'-GAGAGCATTGTCTGCGGGCGATCTTGAGGCCTTCCAG-3'

mrpyr5F 5'-CTGGAAGGCCTCAAGATCGCCCGCAGACAATGCTCTC-3'

mrpyr5R 5'-GACAAAATCCTCGCTCTCCTC-3'

mrarg3F1 5'-CGGTATTGACTAAAAGGGATCTGGAAGGCCTCAAGATCGC-3'

mrarg3F2 5'-CGGTATTGACTAAAAGGGATCCGCTGCAGGCCATC-3'

mrarg3F3 5'-CGGTATTGACTAAAAGGGATGCGCCATCTGCTCTCG-3'

mrarg3R 5'-GAAGGTCAAGGCCGGTG-3'

mrpyr3F 5'-TGCCCCTCCAGGATAAC-3'

mrpyr3R1 5'-GCGATCTTGAGGCCTTCCAGATCCCTITTAGTCAATACCG-3'

mrpyr3R2 5'-GATGGCCTGCAGCGGATCCCTITAGTCAATACCG-3'

mrpyr3R3 5'-CGAGAGCAGATGGCGCATCCCTITAGTCAATACCG-3'

Primers used to amplify DNA probes for Southern blot analyses

ku80R4 5'-GGAACCCAGAAGAATACGACAC-3' 
Table 1 List of primers used in this study (Continued)

\begin{tabular}{ll}
\hline AaargB3f3 & 5'-GAGTGGAAATGTGTAACGGATTGCATGGACCCGAG-3' \\
AaargB3r & 5'-GGGGACATGGCTTCCTAC-3' \\
DxInR-F1 & 5'-GTCGACCACCGCGATCCAGCAGTACGCCA-3' \\
DxInR-R2 & 5'-CACAATCCACAGTGAGACCCACTCTCTCGC-3' \\
\hline
\end{tabular}

The $A$. aculeatus ornithine carbamoyltransferase gene (AaargB) was also cloned from the A. aculeatus genome by the same strategy. The AaargB segment was amplified with the primers AaargBF3 and AaargBR2. After performing Southern blot analysis and colony hybridization using the AaargB segment as a DNA probe, the AaargB open reading frame (ORF) with flanking regions, an approximately $6-\mathrm{kb}$ HindIII-digested fragment, was cloned into pBS II KS (+), yielding pAaargB.

\section{Transformation of $A$. aculeatus}

The transformations were performed by following the protoplast-PEG method as described previously (Adachi et al. 2009). The strains were propagated at $30^{\circ} \mathrm{C}$ in $\mathrm{MM}$ supplemented with the appropriate nutrients.

\section{Gene disruption and marker recycling}

The linear DNA fragments used for the gene deletion of Aaku 80 followed by marker recycling were generated by fusion PCR as described previously (Akada et al. 2006). To construct the Aaku80 deletion cassette, the 5' and 3' regions of the Aaku80 gene, which are required for HR, were amplified from $A$. aculeatus genomic DNA using the primer pairs aku80AF1 and aku80AR2 for the $5^{\prime}$ region and aku80BF1 and aku80BR1 for the 3' region. The 3 ' flanking region of the Aaku80 gene, which formed DRs, was amplified from $A$. aculeatus genomic DNA using the primer pair aku80CF1 and aku80CR1. The AnpyrG fragment was amplified using pPL6 (Punt et al. 1991) as a template with the primer pair npyrF2 and npyrR1. The four fragments were joined by fusion PCR using the primers aku80AF1 and aku80BR1, and the fused product was subcloned into the EcoRV site of pBS II KS (+), which yielded pDku80. The Aaku80 deletion cassette was amplified with the primers aku80AF1 and Aku80BR1 using pDku80 as a template (Figure 1). The fragments were introduced into the pyrG13 strain using the protoplast-PEG method. The deletion of endogenous Aaku 80 was confirmed by Southern blot analysis using DNA probes that were amplified with the primers ku80R4 and aku80BR1 using pAaku80 as a template. FOA-resistant strains by marker recycling were spontaneously isolated from $1 \times 10^{4}$ to $10^{6} \mathrm{~A}$. aculeatus transformant conidia in $\mathrm{MM}$ with $1 \mathrm{mM}$ FOA and $0.4 \mathrm{mM}$ uridine. These conidia were purified by repeating monospore isolation twice on the plates to obtain the conidia of homokaryons. Their growth was examined on plates with or without $8 \mathrm{mM}$ uridine.

To develop a strain enabling a reversible impairment of Aaku80, an Aaku80 disruption cassette 2 was constructed. The 5'- and 3'-regions of the Aaku80 gene were amplified from $A$. aculeatus genomic DNA using the primer pairs coku80NF and coku80NR for the 5' region a

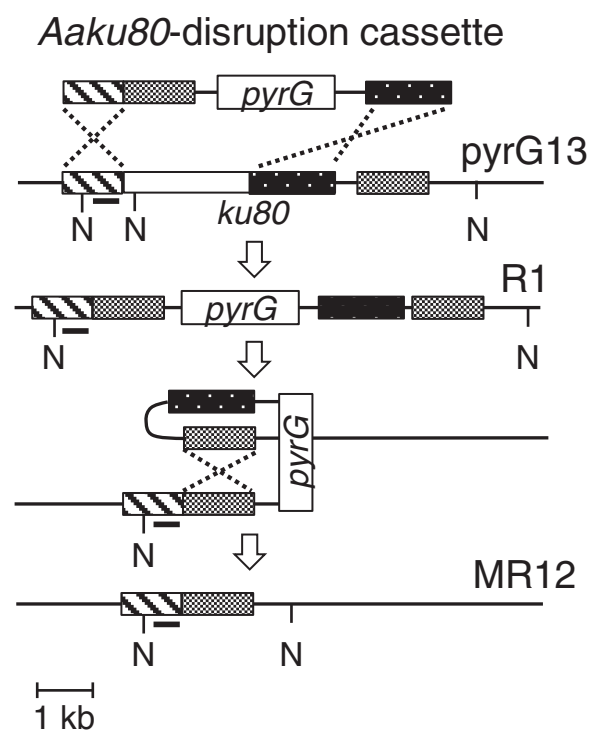

b

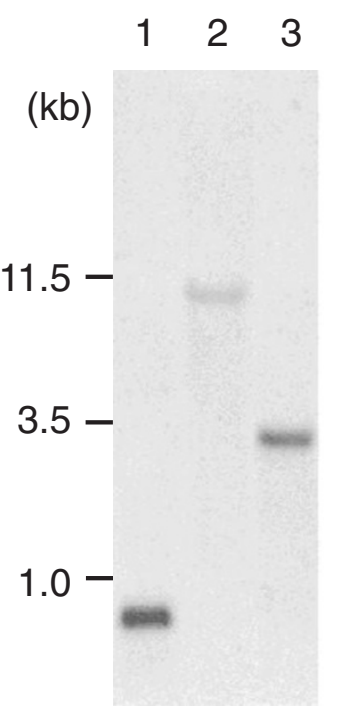

Figure 1 Southern blot analysis of the Aaku80-deletion mutants. (a) Restriction enzyme maps of Aaku80 loci in the pyrG13, R1, and MR12 strains of Aspergillus aculeatus. The DNA probe used for Southern blot analysis is indicated with solid bars. N, restriction sites of Ncol. (b) Results of the Southern blot analysis using genomic DNA digested with Ncol (N). Lanes 1, 2, and 3 are pyrG13, R1, and MR12, respectively. 
and coku80CF and coku80CR for the 3' region. The second intronic region was amplified with the coku80NR and coku80CF primers. The AnpyrG fragment was amplified using pPL6 as a template with the primer pair copyrF and copyrR. The 5'-side of Aaku80 digested with EcoRV and NotI and AnpyrG digested with NotI were inserted into the EcoRV site in pBS II KS (+) to yield pCO5P. The 3'-side of Aaku80 digested with PacI, and $X b a \mathrm{I}$ was ligated to the PacI and XbaI sites on pCO5P to yield pCO5P3. The Aaku80 disruption cassette 2 was amplified with the primers coku80NF and coku80CR using pCO5P3 as a template. The fragments were introduced into the pyrG13 strain using the protoplast-PEG method. The disruption of endogenous Aaku80 was confirmed by Southern blot analysis using DNA probes that were amplified with the primers ku80R4 and aku80BR1 using pAaku80 as a template.

Homologous recombination frequencies were determined by simply replacing the AaargB gene with AnpyrG in pyrG13 and the $A$. aculeatus ku80 deletion-mutant (MR12). To construct the $\operatorname{AaargB}$ deletion cassette 1, the 5' (981 bp) and 3' (1,034 bp) regions of the AaargB gene were amplified from $A$. aculeatus genomic DNA using the primer pairs AaargB5F and AaargB5r2 for the 5' region and AaargB3f3 and AaargB3r for the 3' region. The AnpyrG fragment was amplified using pPL6 as a template with the primer pair npyrF3 and npyrR3. The three fragments were joined by fusion PCR using the primers AaargB5f and AaargB3r, and the fused product was subcloned into the EcoRV site of pBS II KS (+) to yield pDAaargB. The AaargB deletion cassette 1 was amplified with the primers AaargB5f and AaargB3r using pDAaargB as a template. The deletion of endogenous $\operatorname{Aaarg} B$ was confirmed by Southern blot analysis using DNA probes that were amplified with the primers AaargB3f3 and AaargB3r using pAaargB as a template.

To retain the $A a \arg B$ sequence in the arginine auxotroph, the $A \operatorname{aarg} B$ function was disrupted by insertion mutagenesis. This mutation was introduced by overlapping two fragments amplified by primer sets mrarg $5 \mathrm{~F}$ and mrarg5iR and mrarg5if and mrarg5R. These fragments were fused to the 5'-side of the AnpyrG fragment (-456 to 683 nt from the translation start site of AnpyrG) amplified with primer set mrpyr5F and mrpyr5R to yield the 5'arg-pyr fragment. The 3'-side of AaargB fragments with different DR lengths were amplified with the primer sets mrarg3F1 and mrarg3R, mrarg3F2 and mrarg3R, and mrarg3F3 and mrarg3R, respectively. These fragments were fused to the 3'-side of the AnpyrG fragment (174 to $925 \mathrm{nt}$ from the translation start site) amplified with the primer sets mrpyr3F and mrpyr3R1, mrpyr3F and mrpyr3R2, and mrpyr3F and mrpyr3R3 to yield the $\operatorname{argB} 3 \mathrm{~B} 1, \operatorname{argB} 3 \mathrm{~B} 2$, and $\operatorname{argB} 3 \mathrm{~B} 3$ fragments, respectively. In the last step, the 5'argpyr fragment was fused to the $\operatorname{argB} 3 \mathrm{~B} 1, \operatorname{argB} 3 \mathrm{~B} 2$, and
argB3B3 fragments by amplifying with the primer set mrarg5F and mrarg3R, which yielded the argBDR-20, argBDR-98, and argBDR-495 fragments, respectively. These fragments were introduced into the pyrG13 strain using the protoplast-PEG method. The disruption of endogenous AaargB was confirmed by Southern blot analysis using DNA probes that were amplified with the primers mrarg5F and mrarg5iR for the 5'-DNA probe and mrarg3F1 and mrarg3R for the 3'-DNA probe using pAaargB as a template.

Deletion of the $A$. aculeatus $x \ln R$ gene, encoding a gene-specific activator controlling cellulase and hemicellulase gene expression, was performed as described previously (Tani et al. 2012). The $x \ln R$ deletion in the selected strains was confirmed by analyzing each genome using Southern blot analysis and PCR using the primers DxlnRF1 and DxlnR-R2.

\section{Results}

Deletion of the Aaku80 gene and recycling of the AnpyrG marker

As a first step toward developing HR and marker-recycling systems in A. aculeatus, Aaku80 was cloned. Sequence analysis and a homology search revealed that the Aaku80 gene comprised a 2,711-bp ORF interrupted by nine introns and encoding 726 amino acid residues with a calculated molecular mass of 81,088 Da (DDBJ Acc. no. AB741874). The Aaku80 gene in pyrG13 (Aaku80 $\left.{ }^{+}, p y r G^{-}\right)$ was replaced with AnpyrG using the Aaku80 deletion cassette (Figure 1a). Forty transformants were obtained by selection on a uridine-free medium. Southern blot analysis confirmed that one (2.5\%) of these transformants, designated R1, had one copy of the deletion cassette inserted into the Aaku80 locus (Figure 1b, lane 2). Next, we tried to excise the AnpyrG-containing fragment sandwiched by a pair of 1-kb DRs to reuse AnpyrG as a selection marker, as is commonly done in Saccharomyces cerevisiae (Akada, et al. 2006). Conidia (approximately $1 \times 10^{4}$ ) were spread onto a 5-FOA plate, and 20-30 colonies on average that should have been 5-FOA-resistant appeared after $5 \mathrm{~d}$ of cultivation, while it took more than $10 \mathrm{~d}$ for resistant mutants to emerge on 5-FOA plates by spontaneous mutation. After mono-spore isolation, more than $90 \%$ of the isolates showed uridine auxotrophy. Southern blot analysis showed that the expected excision event of intramolecular HR occurred in 9 of 10 uridine auxotrophs (Figure 1b, lane 3). One strain $\left(A a k u 80^{-}, p y r G^{-}\right)$was designated as strain MR12.

\section{Characteristics of the Aaku80 disruptant}

The growth and sporulation of MR12 on MM were indistinguishable from those of the control strain (pyrG13), but its growth on $0.05 \%$ methyl methanesulfonate (MMS) was poorer than that of pyrG13 (Figure 2). This result agreed well with previous reports that $k u$ disruption in Neurospora 


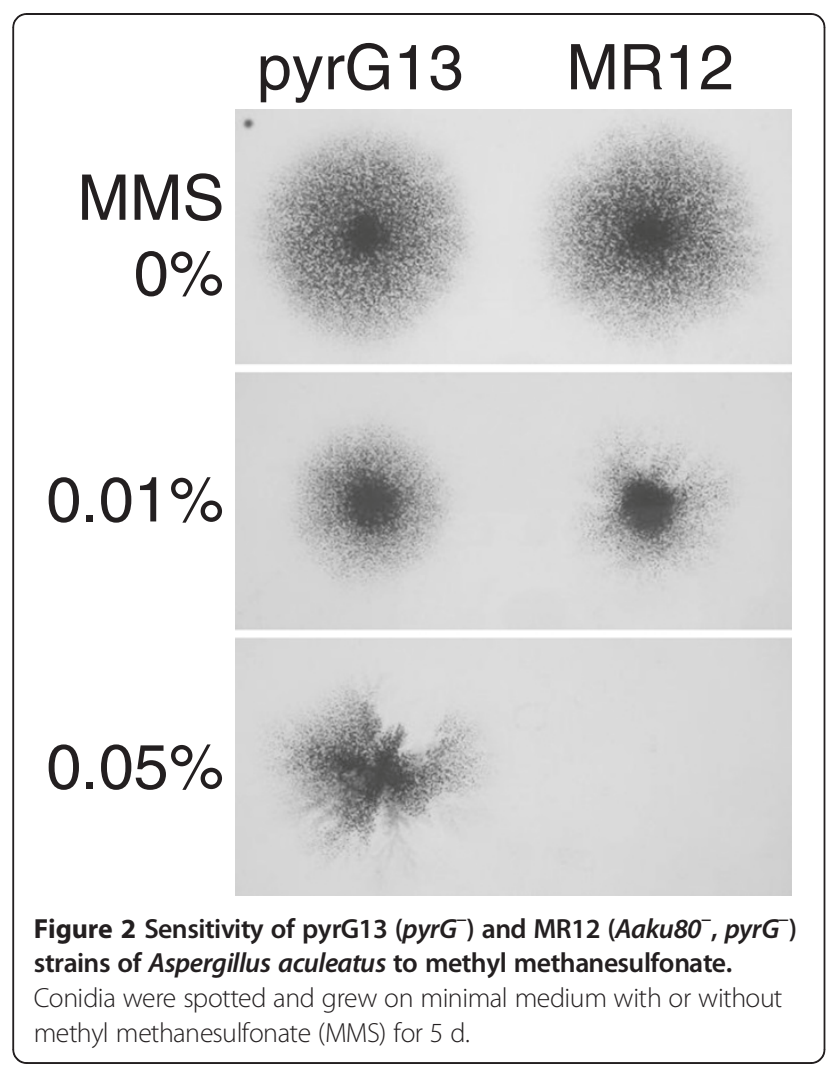

crassa or Aspergillus fumigatus increased sensitivity to the DNA-damaging reagent (Ninomiya et al. 2004; da Silva Ferreira et al. 2006).

To compare the HR frequency at the AaargB locus in pyrG13 with that in MR12, the AaargB gene was cloned. Sequence analysis and a homology search revealed that AaargB comprised a 1,089-bp ORF with no introns and encoded 362 amino acid residues with a calculated molecular mass of 38,980 Da (DDBJ Acc. no. AB741875). The $A \operatorname{aarg} B$ gene was replaced with a selection marker using the AaargB deletion cassette 1 in pyrG13 and MR12 to elucidate the gene targeting frequencies. Arginine auxotrophs were found in one of 32 transformants of pyrG13 (targeting frequency of 3\%) and in 29 of 31 transformants of MR12 (94\%). Southern blot analysis showed that all arginine auxotrophs derived from MR12 had a single copy of the cassette at the target locus by double HR (data not shown).

\section{Construction of pyrG argB double-auxotrophic host and effect of DR length on intramolecular homologous recombination}

The arginine auxotrophic host was generated with point mutations to retain the $\arg B$ ORF as the target of homologous transformation. The $\arg B$ disruption cassette 2 was designed to insert the nucleotides $\mathrm{G}$ and $\mathrm{A}$ behind the 25th $G$ and 27th $T$ from a translation start site, which yielded a NheI recognition site and Ala9Gly mutation followed by a stop codon, respectively (Figure 3a). The disruption cassettes were also designed to harbor different length DRs (20, 98, or $495 \mathrm{bp}$ ) and the AnpyrG selection marker to elucidate the effect of DR length on intramolecule HR. The cassettes were introduced into MR12 and yielded 15, 10, and 20 uridine prototrophic transformants, respectively. From these, 11 (73\%), 10 (100\%), and 17 (85\%) arginine auxotrophs were isolated, respectively. Strains having a single copy of the insert were selected to give the mrTA20, mrTA98, and mrTA495 strains harboring the DRs of the indicated lengths (Figure 3b, lanes 2-4). Conidia $\left(1 \times 10^{6}\right)$ of mrTA495 were spread on plates containing 5-FOA, uridine, and arginine, and 120 colonies developed. Ten 5-FOA-resistant strains were selected for further analysis and found to be uridine auxotrophs. Southern blot analysis confirmed that AnpyrG was excised by intramolecular HR from the $\arg B$ locus in nine of the ten uridine auxotrophs (Figure 3c, lanes 2-4). Thus, an $A$. aculeatus host with double selection markers was successfully constructed. The frequency of the emergence of the uridine auxotroph by intramolecular HR from mrTA495 was calculated to be $1.1 \times 10^{-4}$. Those in mrTA98 and mrTA20 were calculated to be $7.0 \times 10^{-6}$ and $3.0 \times 10^{-6}$, respectively (Table 2). These results indicated that excision frequency was correlated with longer DRs. Although the frequency of AnpyrG excision in mrTA20 was almost equal to the frequency of isolating the uridine auxotroph by spontaneous mutation from the wild-type strain, these events could be distinguished by culture duration, because the 5FOA resistant strains resulting from intramolecular HR appeared earlier than did those by spontaneous mutations.

\section{Mitotic stability analysis of DNA with direct repeat sequences}

Subculturing the mrTA495 strain five times on MM with uridine did not increase the frequency of emergence of 5FOA resistants (data not shown). Furthermore, intramolecular recombination between DRs was investigated by Southern blot analysis using chromosomal DNA isolated from the mrTA495 strain grown in liquid MM with or without uridine for $5 \mathrm{~d}$. The hybridization patterns of the AaargB locus in the mrTA495 were the same as shown in Figure $3 \mathrm{~b}$ (lane 4). The same results were obtained in mrTA20 (lane 2) and mrTA98 (lane 3). These data indicated that the short DRs were stably maintained in the transformants.

\section{Insertion of AnpyrG into the second intron of Aaku80 enables the disruption and restoration of Aaku80}

Establishing a simple method to restore the function of Aaku80 after deleting specific genomic regions would be an advantage in filamentous fungi without a feasible genetic segregation technique, such as $A$. aculeatus. Therefore, we designed the Aaku80 disruption cassette 2, which introduced 


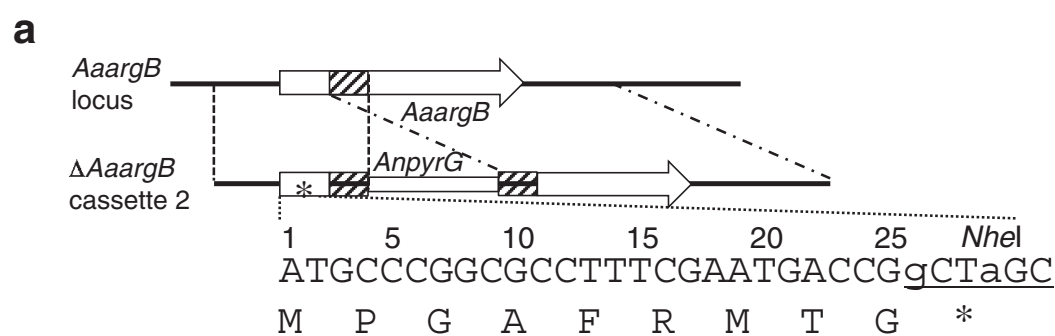

b

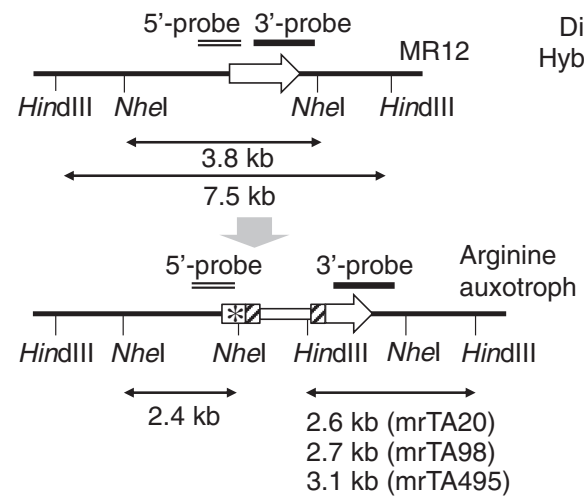

Digested with: Nhel Hybridized with: 5'-probe
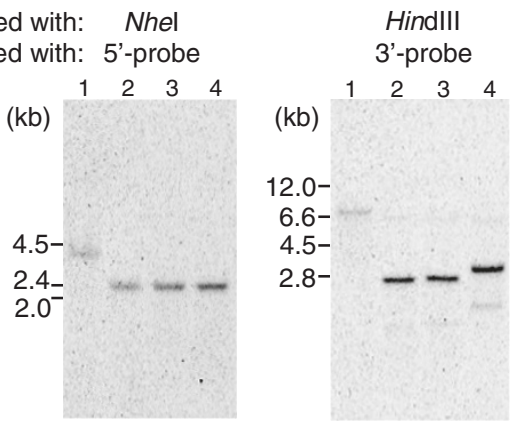

C

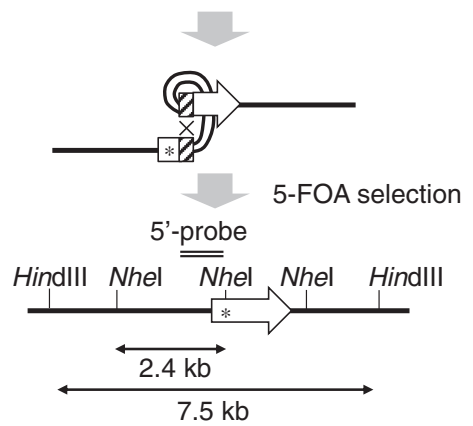

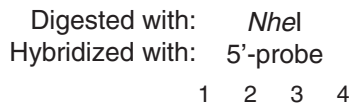

(kb)

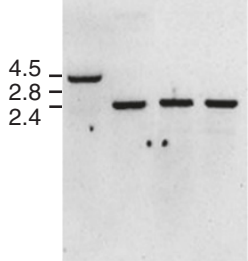

HindIII

5'-probe

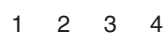

$(\mathrm{kb})$

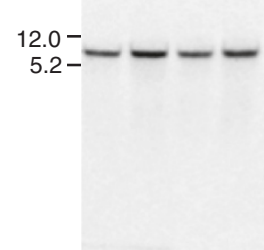

Figure 3 Southern blot analyses of the $\arg B$-auxotroph mutants harboring different lengths of direct repeats for marker recycling. (a) Diagram of the AaargB disruption cassette 2. Thecandy-striped bar indicates the direct repeat sequences. The insertion of guanine $(\mathrm{g})$ and adenine (a) yielded, respectively, a Nhel restriction site and a stop codon (asterisk). (b) Left: restriction enzyme maps of the AaargB loci in the MR12 strain and its transformants with the AaargB disruption cassette 2. The DNA probes used for Southern blot analysis are indicated with a double line (5'-probe) and a solid bar (3'-probe). Right: results of Southern blot analysis using genomic DNA digested with Nhel and Hindlll. Lanes 1, 2, 3, and 4 are MR12, mrTA20, mrTA98, and mrTA495, respectively. (c) Left: restriction enzyme maps of the AaargB locus after the pyrG excision by intramolecular homologous recombination. Right: Southern blot analyses were performed as in (b). Lanes 1, 2, 3, and 4 are MR12 and FOA-resistant strains from mrTA20, mrTA98, and mrTA495, respectively.

the AnpyrG gene into the second intron of the Aakuso gene (Figure 4a). Because the direction of AnpyrG transcription is the same as that of Aaku80, we presumed that the termination of AnpyrG transcription yielded immature transcripts of Aaku80 and resulted in the loss of Ku80 function. Furthermore, we expected that a functional Ku80 protein would be produced even if a frameshift mutation occurred between the repeats because the insert was in an intron in Aaku80. The pyrG13 strain was transformed with the Aaku 80 disruption cassette 2 and yielded transformants harboring the cassette integrated as a single copy, namely coku80, into the Aaku80 locus by homologous integration (Figure 4b, lane 2). The coku80rec strain was isolated by excision of the AnpyrG gene by marker recycling at the frequency of $3.3 \times 10^{-5}$ (Figure $4 \mathrm{~b}$, lane 3 ). Sequence analyses of Aaku80 of five independently-isolated coku80rec strains confirmed that AnpyrG was excised between DRs without any mutations.

Growth and sporulation of the coku80 and coku80rec strains on MM were indistinguishable from those of MR12 
Table 2 Frequencies of intramolecular homologous recombination between direct repeats (DRs) of different lengths

\begin{tabular}{lccc}
\hline Strain & mrTA20 & mrTA98 & mrTA495 \\
\hline Length of DR (bp) & 20 & 98 & 495 \\
No. of 5-FOA-resistant strains & 6 & 14 & 120 \\
No. of resistant strains analyzed & 6 & 6 & 10 \\
No. of uridine auxotrophs & 6 & 4 & 9 \\
No. of homologous & 3 & 3 & 9 \\
recombination events between DRs & & & \\
\hline
\end{tabular}

The 5-fluoroorotic acid (5-FOA) resistant strains were isolated from $1.0 \times 10^{6}$ conidia.

$\left(\right.$ Aaku80-, pyrG ${ }^{-}$) and pyrG13 (pyrG $\left.{ }^{-}\right)$, but growth of coku80 on $0.05 \%$ MMS was as poor as that of MR12. However, both coku80rec and pyrG13 grew normally on $0.05 \%$ MMS (data not shown). Transformation of pyrG13, MR12, coku80, and coku80rec with the $x \ln R$ deletion cassette yielded 1 (of 51 transformants; $2 \%$ ), 31 (of 46; 67\%), 26 (of 35; 74\%), and 1 (of 38; 3\%) xylan-utilization-deficient mutants. AnpyrG insertion into the second intron of Aaku80 successfully disrupted Aaku80 function, which was restored by intramolecular HR between 20-bp DRs. Furthermore, the coku80 strain stably maintained the AnpyrG gene after 10 generations and yielded 17 xylan-utilizationdeficient mutants out of 21 transformants (81\%). These data

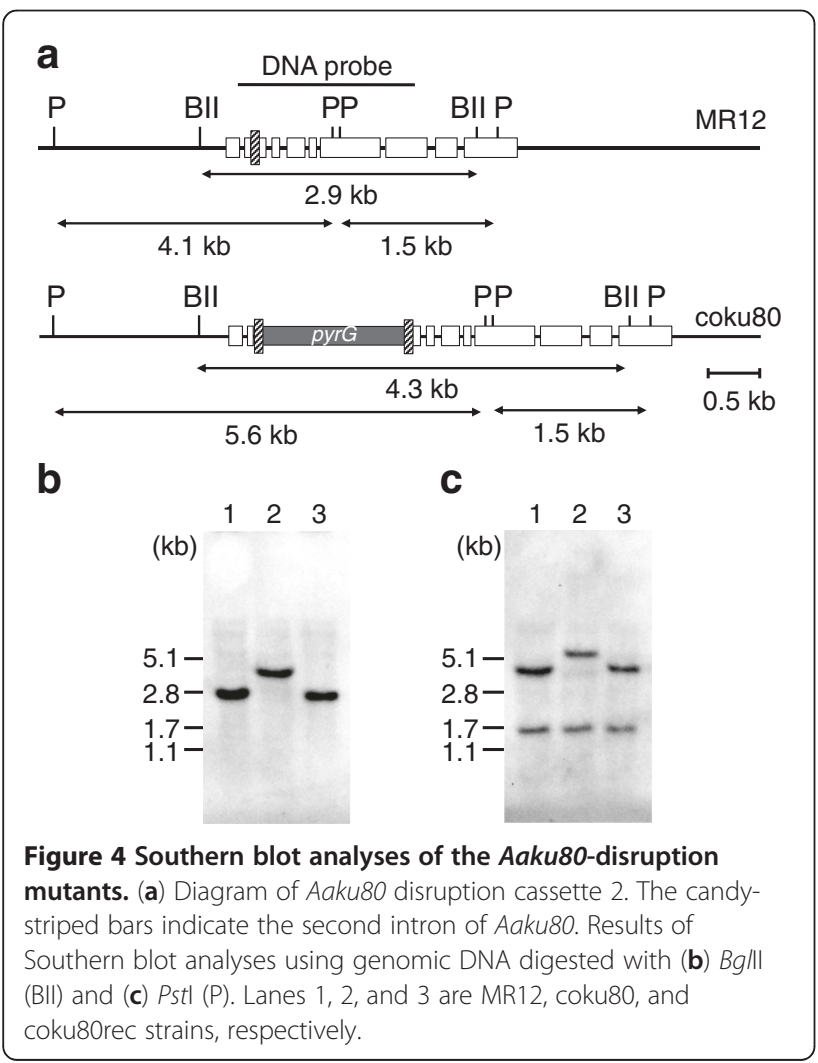

indicate that coku80 is a feasible host for genetic analysis of interesting genes in the Aaku 80 functional background.

\section{Discussion}

In Aspergillus, auxotrophic mutants, such as pyrG, niaD (nitrate reductase), or $s C$ (ATP-sulfurylase)-deficient mutants, can be simply isolated because they are positively selected in the presence of the counter-selective compounds (Arst 1968; Daboussi et al. 1989; Goosen et al. 1987). To advance molecular biology in A. aculeatus, we designed and validated a HR method for gene replacement by deleting the Aaku80 gene. The Aaku80 deletion drastically increased the gene-targeting frequency from $3 \%$ to 94\% and enabled the construction of double auxotrophic mutants (Aaku80, $\operatorname{argB} B^{-}$, pyrG $\left.{ }^{-}\right)$. This drastic increase in gene-targeting frequency reduced the labor required to isolate auxotrophic mutants, such as an arginine auxotroph, without the use of counter-selective compounds. The $94 \%$ targeting frequency in $A$. aculeatus was high enough for practical use in gene replacement. However, the targeting frequency for approximately 1-kb homologous sequences was lower than in $N$. crassa (Ninomiya et al. 2004). Because homology length is critical to the homologous integration frequency, $A$. aculeatus might require a longer homologous region for gene targeting.

Because the number of marker genes is limited, establishing a marker-recycling technique is necessary for the consecutive insertion of gene expression cassettes or multiple-gene deletions. For marker rescue, the cre-loxP recombination system of bacteriophage $\mathrm{P} 1$ has been shown to mediate efficient recombination between 34-bp loxP sites flanking a marker gene in yeast and filamentous fungi, resulting in excision of the marker gene (Krappmann et al. 2005; Sauer 1987). Gene deletion by HR between the loxP sites results in marker removal, leaving behind a single repeat at the deleted gene locus. Therefore, developing a self-cloning technique to breed genetically-modified filamentous fungi is important. To achieve this aim, the double-marker enrichment technique was adopted for target-gene deletion in A. fumigatus, Aspergillus awamori, A. nidulans, and Leptosphaeria maculans (d'Enfert 1996; Gardiner and Howlett 2004; Krappmann and Braus 2003; Michielse et al. 2005). In this strategy, a second selectable marker is added to the gene deletion cassette to distinguish between HR and non-homologous recombination. Upon $\mathrm{HR}$, the second selection marker is lost, whereas after integration by non-homologous recombination, transformants carrying both selectable markers are obtained. Alternatively, once a mutant with a two-way (positive and negative) selectable marker such as pyrG is isolated, marker recycling enables not only consecutive gene deletion, but also consecutive gene insertion without leaving behind an exogenous DNA fragment. Although 50-100 bp of DRs were sufficient to facilitate gene-replacement cassette integration at the 
homologous locus at a gene replacement efficiency of 50$100 \%$ in Schizosaccharomyces pombe and Saccharomyces cerevisiae (Bahler et al. 1998; Wach et al. 1994), longer DRs were thought to be required in filamentous fungi (Hynes 1996; Maruyama and Kitamoto 2008; Nielsen et al. 2008; Ninomiya et al. 2004). However, our results indicated that the correct excision event could occur even with a 20-bp DR, although the frequency was relatively low. The observation that a shorter DR works sufficiently for correct excision should help to design a deletion cassette for marker recycling. Using this method, we are constructing an $A$. aculeatus host with multiple selectable markers to enable the breeding of filamentous fungi that produce several different cellulases to hydrolyze cellulosic substances with high efficiency.

Despite whole-genome sequencing, the roles of many gene products in fungi remain unknown, in part because gene deletion may not alter the phenotype when more than one paralog exists in the genome. The technique developed in this paper could be a useful tool for postgenomic research.

\section{Competing interests}

The authors declare that they have no competing interests.

\section{Acknowledgements}

This work was supported by research grants from the Noda Institute for Scientific Research, Japan.

\section{Author details}

${ }^{1}$ Graduate School of Life and Environmental Sciences, Osaka Prefecture University, 1-1 Gakuen-cho, Naka-ku, Sakai, Osaka 599-8531, Japan. ${ }^{2}$ Present address: Industrial Research Institute of Ishikawa, 2-1 Kuratsuki, Kanazawalshikawa 920-8203, Japan.

Received: 4 September 2012 Accepted: 29 December 2012 Published: 12 January 2013

\section{References}

Adachi H, Tani S, Kanamasa S, Sumitani J, Kawaguchi T (2009) Development of a homologous transformation system for Aspergillus aculeatus based on the $S C$ gene encoding ATP-sulfurylase. Biosci Biotech Biochem 73:1197-11992

Akada R, Kitagawa T, Kaneko S, Toyonaga D, Ito S, Kakihara Y, Hoshida H, Morimura S, Kondo A, Kida K (2006) PCR-mediated seamless gene deletion and marker recycling in Saccharomyces cerevisiae. Yeast 23:399-405

Arst HNJ (1968) Genetic analysis of the first steps of sulphate metabolism in Aspergillus nidulans. Nature 219:268-270

Bahler J, Wu J, Longtine M, Shah N, McKenzie A, Steever A, Wach A, Philippsen P, Pringle J (1998) Heterologous modules for efficient and versatile PCR-based gene targeting in Schizosaccharomyces pombe. Yeast 14:943-951

Daboussi M, Djeballi A, Gerlinger C, Blaiseau P, Bouvier I, Cassan M, Lebrun M, Parisot D, Brygoo Y (1989) Transformation of 7 species of filamentous fungi using the nitrate reductase gene of Aspergillus nidulans. Curr Genet 15:453-456

d'Enfert C (1996) Selection of multiple disruption events in Aspergillus fumigatus using the orotidine- $5^{\prime}$-decarboxylase gene, $p y r G$, as a unique transformation marker. Curr Genet 30:76-82

Gardiner DM, Howlett BJ (2004) Negative selection using thymidine kinase increases the efficiency of recovery of transformants with targeted genes in the filamentous fungus Leptosphaeria maculans. Curr Genet 45:249-255

Goosen T, Bloemheuvel G, Gysler C, Debie D, Vandenbroek H, Swart K (1987) Transformation of Aspergillus Niger using the homologous orotidine5'-phosphate decarboxylase gene. Curr Genet 11:499-503

Hynes M (1996) Genetic transformation of filamentous fungi. J Genet 75:297-311
Ishibashi K, Suzuki K, Ando Y, Takakura C, Inoue H (2006) Nonhomologous chromosomal integration of foreign DNA is completely dependent on MUS-53 (human Lig4 homolog) in Neurospora. Proc Natl Acad Sci USA 103:14871-14876 Kanamasa S, Yamaoka K, Kawaguchi T, Sumitani J, Arai M (2003) Transformation of Aspergillus aculeatus using the drug resistance gene of Aspergillus oryzae and the pyrG gene of Aspergillus nidulans. Biosci Biotechnol Biochem 67:2661-2663

Kanamasa S, Kawaguchi T, Takada G, Kajiwara S, Sumitani J, Arai M (2007) Development of an efficient production method for $\beta$-mannosidase by the creation of an overexpression system in Aspergillus aculeatus. Lett Appl Microbiol 45:142-147

Krappmann S, Braus GH (2003) Deletion of Aspergillus nidulans aroC using a novel blaster module that combines ET cloning and marker rescue. Mol Gen Genomics 268:675-683

Krappmann S, Bayram Ö, Braus GH (2005) Deletion and allelic exchange of the Aspergillus fumigatus veA locus via a novel recyclable marker module. Eukaryot Cell 4:1298-1307

Krappmann S, Sasse C, Braus GH (2006) Gene targeting in Aspergillus fumigatus by homologous recombination is facilitated in a nonhomologous end-joining-deficient genetic background. Eukaryot Cell 5:212-215

Maruyama J, Kitamoto K (2008) Multiple gene disruptions by marker recycling with highly efficient gene-targeting background $(\Delta / \mathrm{ig} D)$ in Aspergillus oryzae. Biotechnol Lett 30:1811-1817

Michielse C, Arentshorst M, Ram A, van den Hondel C (2005) Agrobacteriummediated transformation leads to improved gene replacement efficiency in Aspergillus awamori. Fungal Genet Biol 42:9-19

Mizutani O, Kudo Y, Saito A, Matsuura T, Inoue H, Abe K, Gomi K (2008) A defect of ligD (human lig4 homolog) for nonhomologous end joining significantly improves efficiency of gene-targeting in Aspergillus oryzae. Fungal Genet Biol 45:878-889

Murao S, Kanamoto J, Arai M (1979) Isolation and identification of a cellulolytic enzyme producing microorganism. J Ferment Technol 57:151-156

Murao S, Sakamoto R, Arai M (1988) Cellulases of Aspergillus aculeatus. Method Enzymol 160:274-299

Nielsen JB, Nielsen ML, Mortensen UH (2008) Transient disruption of nonhomologous end-joining facilitates target genome manipulations in the filamentous fungus Aspergillus nidulans. Fungal Genet Biol 45:165-170

Ninomiya Y, Suzuki K, Ishii C, Inoue H (2004) Highly efficient gene replacements in Neurospora strains deficient for nonhomologous end-joining. Proc Natl Acad Sci USA 101:12248-12253

Punt PJ, Zegers ND, Busscher M, Pouwels PH, Vandenhondel C (1991) Intracellular and extracellular production of proteins in Aspergillus under the control of expression signals of the highly expressed Aspergillus nidulans gpdA gene. J Biotechnol 17:19-33

Sauer B (1987) Functional expression of the cre-lox site-specific recombination system in the yeast Saccharomyces cerevisiae. Mol Cell Biol 7:2087-2096

da Silva Ferreira ME, Kress MR, Savoldi M, Goldman MH, Hartl A, Heinekamp T, Brakhage AA, Goldman GH (2006) The akuB ${ }^{K \cup 80}$ mutant deficient for nonhomologous end joining is a powerful tool for analyzing pathogenicity in Aspergillus fumigatus. Eukaryot Cell 5:207-211

Takahashi T, Masuda T, Koyama Y (2006) Identification and analysis of Ku70 and Ku80 homologs in the Koji molds Aspergillus sojae and Aspergillus oryzae. Biosci Biotech Biochem 70:135-143

Tani S, Kanamasa S, Sumitani J, Arai M, Kawaguchi T (2012) XInR-independent signaling pathway regulates both cellulase and xylanase genes in response to cellobiose in Aspergillus aculeatus. Curr Genet 58:93-104

Wach A, Brachat A, Pohlmann R, Philippsen P (1994) New heterologous modules for classical or PCR-based gene disruptions in Saccharomyces cerevisiae. Yeast 10:1793-1808

doi:10.1186/2191-0855-3-4

Cite this article as: Tani et al.: Reversible impairment of the ku80 gene by a recyclable marker in Aspergillus aculeatus. AMB Express 2013 3:4. 